\title{
EDITORIAL
}

For reprint orders, please contact: reprints@futuremedicine.com

\section{Identifying roadblocks to successful oncolytic virotherapy: what are they and how do we approach them?}
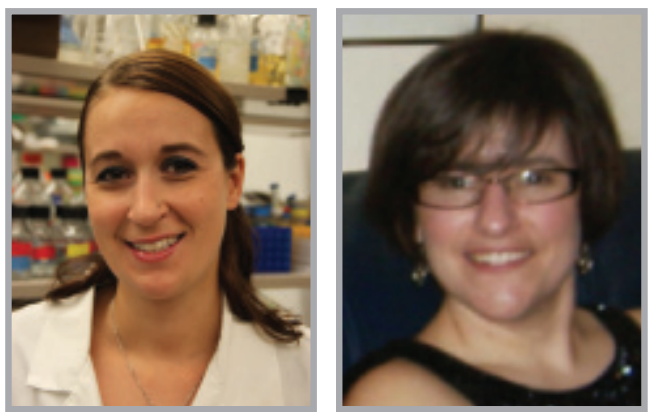

\author{
"The gaps between \\ preclinical and clinical results \\ suggest that we do not fully \\ understand the interactions \\ of oncolytic viruses with host \\ factors."
}

Breanne P Cuddington' \& Karen L Mossman*,1

Simply put, cancer is the collection of gainof-function and loss-of-function mutations that give rise to a tumor or an accumulation of mutated cells. While this definition is succinct, it gives no allusion to the diverse and dynamic nature of the disease. The development of oncolytic viruses (OVs) as cancer therapeutics has accelerated over the past few decades due to the limited success with conventional therapies, such as radio- and chemo-therapy. An increased understanding of virus-host interactions and the impact of the tumor microenvironment and host immune system on oncolytic virotherapy (OVT) has led to clinical progress in the field. Clinical trials have demonstrated the safety and efficacy of OVs in a variety of malignancies. In 2005, a milestone was realized when the first OV (H101) was approved for marketing by Chinese regulators [1]. While there have been tremendous advancements made, the gaps between preclinical and clinical results suggest that we do not fully understand the interactions of OVs with host factors.

Hitting the ground running, the ability of OVs to infect and kill tumor cells is evaluated in vitro, often using highthroughput screening techniques. This approach is useful for characterizing virushost interactions at a basic level and identifying potential model systems going forward. However, recent studies have shown that in vitro assays do not always predict in vivo outcomes $[2,3]$. Tumors are complex organs that contain a plethora of hurdles to both virus replication and tumor clearance that cannot be fully modeled in vitro. That being said, the identification of molecular barriers to oncolysis may aid in the design of an effective therapeutic. Furthermore, the implementation of valid in vivo experimental systems will be required in order to evaluate the safety and efficacy of OVs in a more clinically relevant context. The majority of studies still employs immunodeficient mice with human tumor cell xenografts in order to study therapeutic efficacy. While these models are convenient, not only are they less indicative of clinical practice, but they also fail to take into account the role of the host immune system and orthotopic tumor microenvironment.

There are many obstacles to successful OVT that have been identified. The

\section{KEYWORDS}

- challenges • immunovirotherapy

- oncolytic virotherapy • tumor microenvironment $\bullet$ virus-host interactions 
interactions between tumor cells and their microenvironment play a pivotal role in tumor growth and invasiveness. Arguably, the tumor microenvironment represents the most formidable defense a tumor has against OVT (reviewed in [4]). For instance, the tumor microenvironment can influence the response to OVT by affecting $\mathrm{OV}$ delivery and dissemination, the infiltration and activation of immune cells, bystander killing effects and, ultimately, tumor regrowth. On the other hand, the impact of OVT on the tumor microenvironment varies on a case-by-case basis. OVs can have both direct and indirect pro- and anti-angiogenic effects. Directly, OVs are able to infect and lyse endothelial cells. Indirectly, they can alter the expression of angiogenic factors in order to increase or decrease microvessel density. Furthermore, inflammatory responses to OVT can modulate vascular perfusion, increasing immune cell and cytokine recruitment to the tumor. Thus, OVs are excellent candidates by which to destabilize and increase the immunogenicity of the tumor microenvironment. However, the use of angiostatic regulators remains controversial. While they can be used to increase virus spread by decreasing immune infiltration into the tumor, they can also induce vascular shutdown, leading to tumor cell hypoxia $[5,6]$. This has varied effects depending on the model and OV $[7,8]$. While this may be detrimental to one OV, it may increase the efficacy of another. Furthermore, resident and infiltrating immune cells can be used by the tumor to produce a proangiogenic, tumorigenic and invasive environment. Reprogramming these cells to elicit an antitumor immune response during OVT presents an attractive opportunity for improving therapeutic outcomes by breaking immune tolerance. Presumably, this will result in sustained systemic anti-tumor protection and enhance therapeutic efficacy against metastatic disease.

The dogma that OVs elicit tumor regression due to direct oncolysis of tumor cells has been amended to include a wide repertoire of mechanisms. The host immune system has been identified as the most influential mechanism by which OVs elicit tumor cell death. Recent studies have shown evidence of anti-tumor responses in which regression is observed in injected as well as contralateral uninfected tumors $[9,10]$. In a Phase III clinical trial, an effective and sustained antitumor immune response was observed in melanoma patients receiving talimogene laherparepvec (also known as T-Vec), an oncolytic herpesvirus expressing GM-CSF [11]. Furthermore, the induction of proinflammatory and danger signals potentiates innate and adaptive anti-tumor and antiviral immune responses and contributes to therapeutic efficacy $[2,12,13]$. The involvement of the immune system in OVT is a double-edged sword and must be evaluated within each model system and for each therapeutic regimen. The difficulty facing researchers will be in finding a balance, such that modulation of the immune response will allow for sufficient viral replication and spread while sustaining immune cell infiltration into the tumor, epitope spreading and cross-priming of tumor-associated antigens [6,14]. Recent studies using immunosuppressive drugs have shown increased viral replication and spread, resulting in positive therapeutic outcomes $[15,16]$. Furthermore, the treatment of metastatic disease remains a major hurdle to successful cancer therapy. Engineering OVs to express secretable immunostimulatory molecules represents a novel method by which to induce self-perpetuating and sustained systemic anti-tumoral protection in patients with nonresectable disease [17-19]. Although the process by which OVs mediate anti-tumoral immunity is poorly understood, one thing is clear: their development as an immunovirotherapy to drive anti-tumor immunity will bring them to the front line as a relevant cancer vaccine. Therefore, future studies should include immunological readouts in addition to traditional end points in order to aid in the understanding of the immune-mediated effects of OVs.

OVs have many attributes that make them attractive cancer therapeutics; however, recent studies demonstrate that it is likely that a combination therapy approach will be necessary to achieve a sustained anti-tumor responses. The combination of OVs with traditional treatment modalities, such as radio- and chemo-therapy, has fallen short of this goal, making the identification of novel synergistic combinations critical. Current efforts focused on understanding the interaction of OVs with the tumor microenvironment and host immune system and the roles these factors play in virotherapy have uncovered novel targets for combination therapy. For instance, the impact of OVs on the tumor vasculature has raised interest in combining OVs with antiangiogenic drugs $[5,20]$. Furthermore, targeted disruption of the structural integrity of the tumor microenvironment, which has also been shown to promote viral spread, has a significant impact on tumor biology and can influence responses to 
therapy. We would strongly argue that methods aimed at promoting viral spread should be considered a distant second to those that elicit an antitumor immune response. A multitude of studies demonstrate the importance of the immune system in OVT, such that immunotherapy has come to the forefront as a method by which to achieve prolonged anti-tumor responses, even when the OV has been cleared. In these cases, the OV serves as a primer to break immunological tolerance by increasing epitope spread and inducing cross-priming of tumor-associated antigens. Ultimately, our ability to understand the relationship between an OV and a particular therapeutic will determine the success of combination OVT.

Recent progress in the field fills us with optimism that OVs will find their place as effective cancer vaccines. In our opinion, the future hinges on synergistic combination therapy and an understanding of its effects as they pertain to each model and virus. It is also our firm belief that a more detailed knowledge of virus-host interactions and basic cancer biology will make it possible to adapt viruses for cancer therapy. A back-tobasics approach is needed in which the focus is on gaining an understanding of the agents we work with and how they interact. Only then will we cross the finish line with a winning therapeutic.

\section{Financial \& competing interests disclosure}

BP Cuddington holds a fellowship from the Canadian Breast Cancer Foundation. This work was sponsored by operating grants from the Cancer Research Society and the Canadian Cancer Society Research Institute (formerly the Canadian Breast Cancer Research Alliance). The authors have no other relevant affiliations or financial involvement with any organization or entity with a financial interest in or financial conflict with the subject matter or materials discussed in the manuscript apart from those disclosed.

No writing assistance was utilized in the production of this manuscript.

\section{“...immunotherapy has come to the forefront as a method by which to achieve prolonged anti-tumor responses, even when the oncolytic virus has been cleared."}

\section{References}

1 Garber K. China approves world's first oncolytic virus therapy for cancer treatment. J. Natl Cancer Inst. 98(5), 298-300 (2006).

2 Workenhe ST, Simmons G, Pol JG, Lichty BD, Halford WP, Mossman KL. Immunogenic HSV-mediated oncolysis shapes the antitumor immune response and contributes to therapeutic efficacy. Mol. Ther. 22(1), 123-131 (2014).

3 Workenhe ST, Pol JG, Lichty BD, Cummings DT, Mossman KL. Combining oncolytic HSV-1 with immunogenic cell deathinducing drug mitoxantrone breaks cancer immune tolerance and improves therapeutic efficacy. Cancer Immunol. Res. 1(5), 309-319 (2013).

4 Wojton J, Kaur B. Impact of tumor microenvironment on oncolytic viral therapy. Cytokine Growth Factor Rev. 21(2-3), 127-134 (2010).

5 Libertini S, Iacuzzo I, Perruolo G et al. Bevacizumab increases viral distribution in human anaplastic thyroid carcinoma xenografts and enhances the effects of E1A-defective adenovirus d1922-947. Clin. Cancer Res. 14(20), 6505-6514 (2008).

6 Breitbach CJ, Paterson JM, Lemay CG et al. Targeted inflammation during oncolytic virus therapy severely compromises tumor blood flow. Mol. Ther. 15(9), 1686-1693 (2007).

7 Shen BH, Bauzon M, Hermiston TW. The effect of hypoxia on the uptake, replication and lytic potential of group $B$ adenovirus type $3(\mathrm{Ad} 3)$ and type $11 \mathrm{p}(\operatorname{Ad} 11 \mathrm{p})$. Gene Ther. 13(12), 986-990 (2006).

8 Fasullo M, Burch AD, Britton A. Hypoxia enhances the replication of oncolytic herpes simplex virus in $\mathrm{p} 53$ - breast cancer cells. Cell Cycle 8(14), 2194-2197 (2009).

9 Georgel P, Jiang Z, Kunz S et al. Vesicular stomatitis virus glycoprotein $\mathrm{G}$ activates a specific antiviral Toll-like receptor 4-dependent pathway. Virology 362(2), 304-313 (2007).

10 Toda M, Rabkin SD, Kojima H, Martuza RL. Herpes simplex virus as an in situ cancer vaccine for the induction of specific anti-tumor immunity. Hum. Gene Ther. 10(3), 385-393 (1999).

11 Hersey P, Gallagher S. Intralesional immunotherapy for melanoma. J. Surg. Oncol. 109(4), 320-326 (2014).

12 Heinzerling L, Kunzi V, Oberholzer PA, Kundig T, Naim H, Dummer R. Oncolytic measles virus in cutaneous T-cell lymphomas mounts antitumor immune responses in vivo and targets interferon-resistant tumor cells. Blood 106(7), 2287-2294 (2005).

13 Sobol PT, Boudreau JE, Stephenson K, Wan Y, Lichty BD, Mossman KL. Adaptive antiviral immunity is a determinant of the therapeutic success of oncolytic virotherapy. Mol. Ther. 19(2), 335-344 (2011).

14 Haralambieva I, Iankov I, Hasegawa K, Harvey M, Russell SJ, Peng KW. Engineering oncolytic measles virus to circumvent the intracellular innate immune response. $\mathrm{Mol}$. Ther. 15(3), 588-597 (2007).

15 Jha BK, Dong B, Nguyen CT, Polyakova I, Silverman RH. Suppression of antiviral innate immunity by sunitinib enhances oncolytic virotherapy. Mol. Ther. 21(9), 1749-1757 (2013).

16 Peng KW, Myers R, Greenslade A et al. Using clinically approved cyclophosphamide regimens to control the humoral immune response to oncolytic viruses. Gene Ther. 20(3), 255-261 (2013).

17 Yu F, Wang X, Guo ZS, Bartlett DL, Gottschalk SM, Song XT. T-cell engager-armed oncolytic vaccinia virus significantly enhances antitumor therapy. Mol. Ther. 22(1), 102-111 (2014).

18 Heo J, Reid T, Ruo L et al. Randomized dose-finding clinical trial of oncolytic immunotherapeutic vaccinia JX-594 in liver cancer. Nat. Med. 19(3), 329-336 (2013).

19 Pesonen S, Diaconu I, Kangasniemi L et al. Oncolytic immunotherapy of advanced solid tumors with a CD40L-expressing replicating adenovirus: assessment of safety and immunologic responses in patients. Cancer Res. 72(7), 1621-1631 (2012).

20 Katsura T, Iwai S, Ota Y, Shimizu H, Ikuta K, Yura Y. The effects of trichostatin A on the oncolytic ability of herpes simplex virus for oral squamous cell carcinoma cells. Cancer Gene Ther. 16(3), 237-245 (2009). 